\title{
Betatron Stochastic Cooling in the Debuncher: Present and Future
}

\author{
Vladimir Visnjic \\ Fermi National Accelerator Laboratory \\ P.O. Box 500, Batavia, Illinois 60510
}

July 1993 
TM-1845

July 1993

\title{
Betatron Stochastic Cooling in the Debuncher: Present and Future
}

\author{
Vladimir Visnjic. \\ Fermi National Accelerator Laboratory \\ Batavia, IL 60510
}

\begin{abstract}
A cletailed study of the betation stochastic cooling in the Delnuncher is presented. First, a complete theoretical model including the enittanctdependent signal-to-noise ratio as well as time-dependent mixing is constructed. The emittance measurements in the Debuncher are described and it is shown that the model is in excellent agreement with the experinential data. The idea of gain shaping is proposed and it is shown that the gain shaping would improve the cooling of the beam. Several proposals for fitture improvements are studied and appraised, in particular, gain shaping, ramped $\eta$, and cryogenic and "smart" pickups and kickers. Finally, the demands which the Main Injector will impose on the Debuncher are analyzed and a design of the betatron stochastic cooling system for the Main Injector era is outlined.
\end{abstract}




\section{Introduction}

Presently, the Fermilab Antiproton Debuncher accepts $4 \times 10^{7}$ antiprotons every 2.4 seconds and prepares them for injection into the Accumulator for stacking. Besides the bunch rotation, the stochastic cooling of the antiproton beam is the essential part of this process. The initial rms emitiance of the antiproton beam injected from the target is approximately $7.5 \pi \mathrm{mm}$ mrad, while the emittance of the extracted beam is about $1 \pi \mathrm{mm}$ mad. With the advent of Main Injector, the number of antiprotons injected into the Debuncher is expected to increase threefold, while at the same time the period between injections should decrease to $1.5 \mathrm{sec}$. Since the cooling rate is inversely proportional to the number of particles in the beam, this will inevitably lead to slowing down the rate at, which the antiproton beam is cooled and in addition allow less time for the cooling process. Based upon these observations an upgrade of the stochastic cooling systems in the Debuncher is necessary.

In Section 2, the question of gain optimization in the present system is examined and the notion of gain shaping introduced. A brief description of the measurement results is given in Section 3. It is shown that these results are in excellent agreement with the theoretical model. In Section 4 , the theoretical model is further improved by including the effect of time dependent mixing which is due to the momentum cooling in the Debuncher. Next, a detailed discussion of gain shaping is taken up in Section 5 . In Section 6 , the betatron cooling system equipped with "smart.pickups and kickers whose gap is determined by the emittance of the beam is studied. They offer a dramatic improvement of the performance of the system and will presumably have to be implemented in the Main Injector era.

Section 7 deals with the demands on the Debuncher in the Main Injector era. It is shown that major upgrade of the cooling system will be necessary, and main options for the upgrade are discussed. Finally, an outline is given of what might be the only possible design of the stochastic cooling system for the Main Injector era. 


\section{Which optimal gain?}

The process of betatron cooling is described by the differential equation

$$
\frac{d \epsilon}{d t}=-g \frac{W}{N}(2-g(M+U)) \epsilon,
$$

where $W=f_{\text {max }}-f_{\text {min }}$ is the amplifier bandwidth, $N$ is the number of particles in the beam, $g$ is the system gain, $M$ is the mixing factor, and $l$ is the noise to signal ratio.

The mixing factor is basically the number of turns a particle needs to make in order to leave the original sample. It is thus determined by the amplifier bandwidth, the momentum spread of the beam and the slip factor $\eta$ of the machine,

$$
M=\frac{p_{0} f_{0} \phi(p) \ln \frac{f_{\max . x}}{f_{\min }}}{2 \eta W N}
$$

where $\psi(p)$ is the momentum distribution of the particles, $p_{0}$ the energy of the central orbit, and $f_{0}$ the revolution frequency. The mumerical value of the mixing factor with $\frac{\Delta y}{p}=0.3 \%$ is about 10 . Due to momentum cooling in the Debuncher, the momentum spread is reduced by about a factor of two during the cooling cycle. This time dependence of $M$ is discussed in Section 4.

Since the signal is proportional to the emittance of the beam, while the noise is generated by the electronics hardware, it is convenient to write the noise-to-sigual ratio as

$$
U=\frac{\epsilon_{t h}}{\epsilon},
$$

where the "thermal emitance" $\epsilon_{t h}$ is a hardware parameter which represents the thermal noise of the system. $\epsilon_{t h}$ is given by the formula ${ }^{1}$

$$
\epsilon_{t h}=\frac{2 k\left(T_{R}+T_{a m p}\right) h^{2}}{d^{2} Z_{0} e^{2} f_{0} n_{\eta} N \beta},
$$

where $\mathrm{k}$ is the Boltzmann constant, $T_{R}$ and $T_{a m p}$ the absolute temperaturess of the resistors and the amplifier, $h$ the pickup and kicker gap, $d$ the pickul and kicker sensitivity, $Z_{0}$ pickup and kicker characteristic impedance, $t$ the electron charge, $n_{p}$, the number of pickups and kickers, and $\beta$ the vatule of 
$\beta$ function at the position of pickups and kickers. Usually, $U$ is taken to be constant, in which case the cooling rate is also constant,

$$
\frac{1}{\tau}=g \frac{W}{N}(2-g(M+U))
$$

In the Debuncher, however, the emittance changes by an order of magniturle during the cooling cycle and $U=$ const. is a poor approximation. Because of this, the optimal value of the gain cannot be determined from the coefficients of the differential equation (1) alone. Rather, we have to solve the equation and study the emittance evolution for finite times. As we shall see, due to the rapid change of emittance during the cooling process, the noise term $t_{t h} / \mathrm{c}$ becomes the dominant limiting factor toward the end of the cooling cycle.

The differential equation (1) can be solved analytically and the solution is

$$
\epsilon(g, t)=\left(\epsilon\left(g, t^{\prime}\right)-\frac{g}{2-g M} \epsilon_{t h}\right) \epsilon^{g \frac{W}{N}(2-g M)\left(t^{\prime}-t\right)}+\frac{g}{2-g M} \epsilon_{t h},
$$

where $t^{\prime}$ is an arbitrarily chosen time. Because $f(g, 0)=t(0, t) \equiv t_{01}$ it is convenient to choose $t^{\prime}=0$, i.t.

$$
\epsilon(g, t)=\left(\epsilon_{0}-\frac{g}{2-g M} \epsilon_{t h}\right) e^{-g \frac{W}{N}(2-g M) t}+\frac{g}{2-g M} \epsilon_{t h} .
$$

The three dimensional plot $e(y, t)$ is shown in Fig. 1. The meaning of the optimal gain is now clear: if the gain is too small the time constant

$$
\tau=\left\{g \frac{W}{N}(2-g M)\right\}^{-1}
$$

is large and the cooling is slow; if it is too large, the asymptotic value of the emittance

$$
\epsilon_{\infty}=\frac{y}{2-y M} \epsilon_{t h}
$$

is large, despite the fact that the initial cooling rate may be high. 


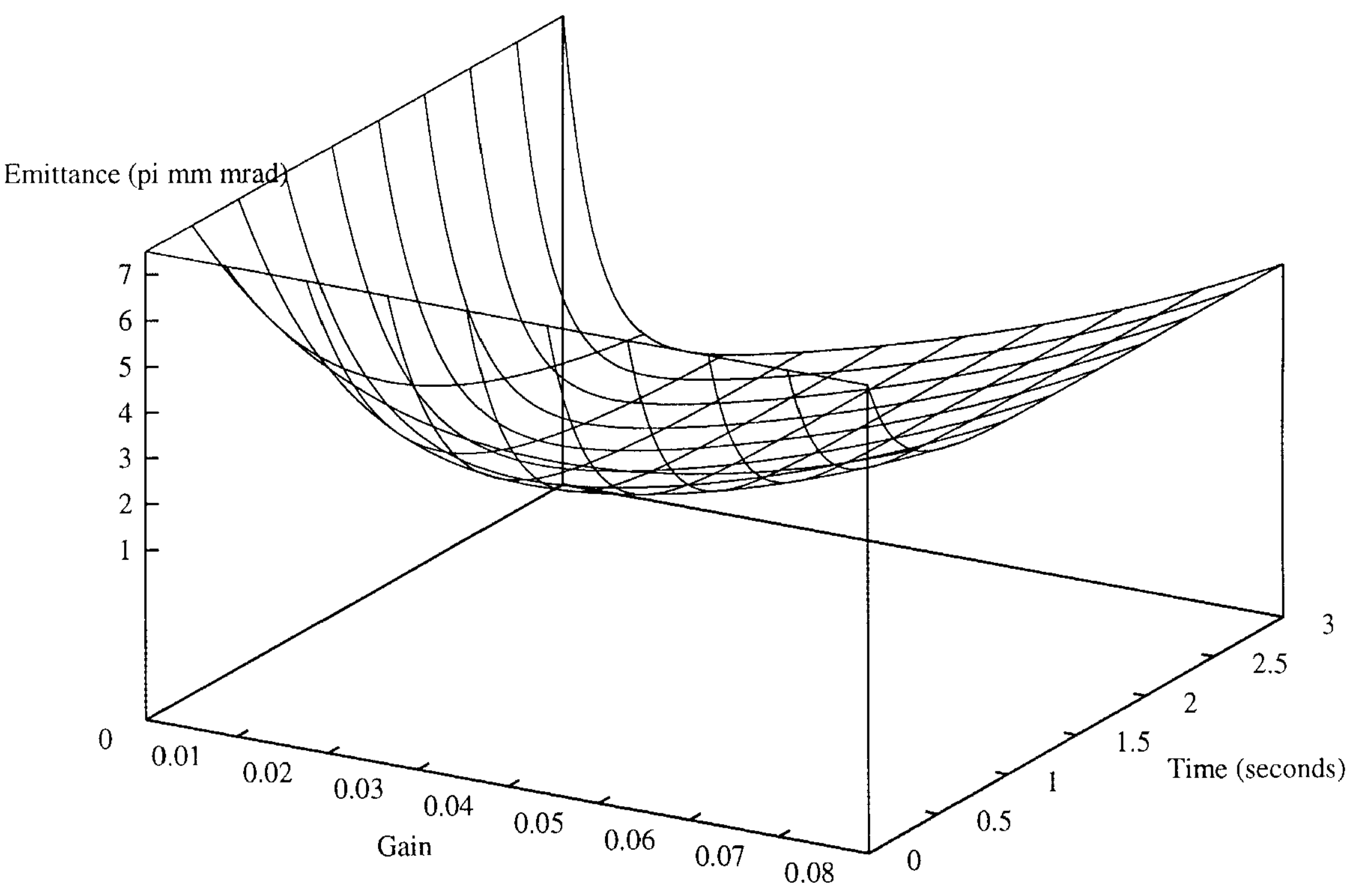

FIGURE 1 The enithance as a function of gain and time. 
In conclusion, the correct clefinition of the optimal gain is:

The optimal gain is the gain which leads to the minimal walue of the rmittance at the end of the cooling cycle:

The optimal gain clearly depends not only on hardware palameters hit also on how long the cooling cycle is - for short cooling cycles we shonkl use a larger gain, for long cooling cycles a smaller gain should be nued. If the cooling cycle has a duration $t_{c}$, the optimal gain is the solution of the equation

$$
\frac{\partial \epsilon\left(g, t_{c}\right)}{\partial y}=0
$$

After some algebra, this equation becomes

$$
N\left(e^{g \frac{W t_{c}}{N}(2-g M)}-1\right) \frac{\epsilon_{t h}}{\epsilon_{0}}=W t_{c}(1-g M)(2-g M)\left(2-g M-g \frac{\epsilon_{t h}}{\epsilon_{0}}\right),
$$

where $\epsilon_{0}=\epsilon(0)$. For the present values of the hardware parameters and the beam intensity, the optimal gain as a function of the duration of the cooling cycle is shown in Fig. 2.

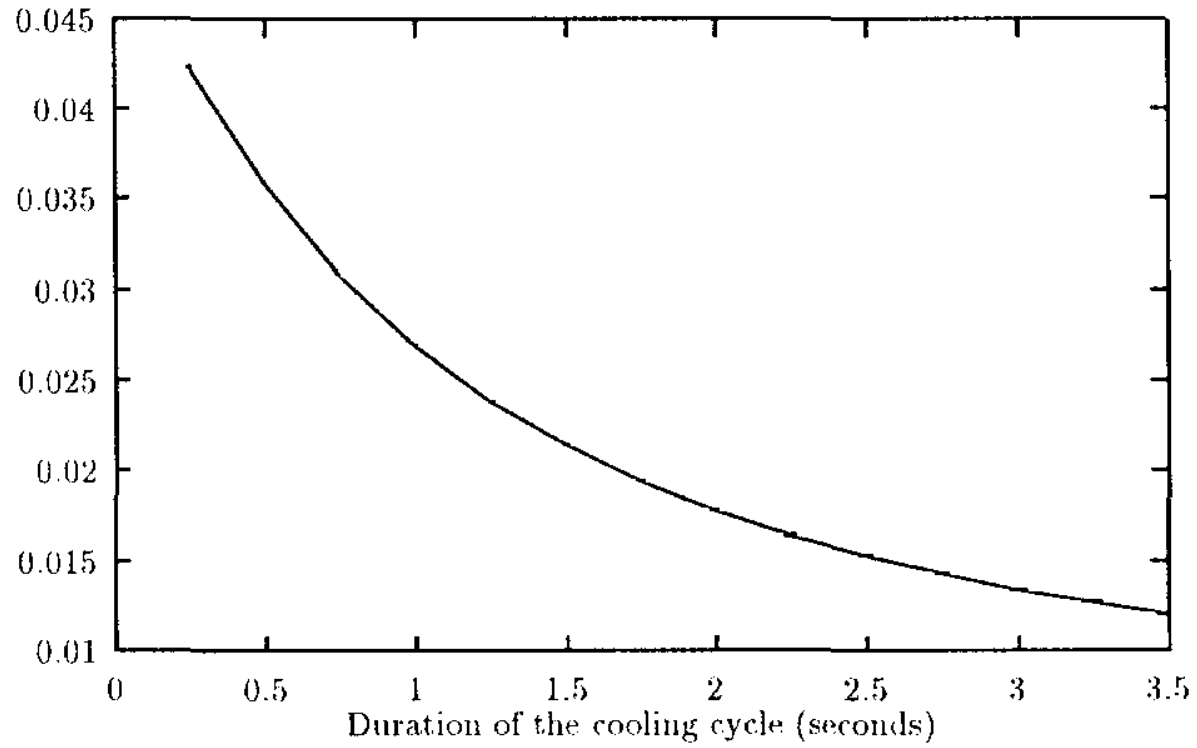

FIGURE 2 The optimal constant gain as a function of the duration of the conling cycle. 
In practice, one deals with power rather than with the gain. If the gain phase is less than $30^{\circ}$ throughout the passband (and in practice it is less than that), the power is approximately proportional to the square of the gain:

$$
P=C y^{2}
$$

where $C$ depends both on hardware and lattice parameters. With this, Eq. (5) becomes

$$
\epsilon(P, t)=\left(\epsilon_{0}-\frac{\sqrt{\frac{P}{C}}}{2-\sqrt{\frac{P}{C}} M} \epsilon_{t h}\right) e^{-\sqrt{\frac{P}{C}} \frac{W}{N}\left(2-\sqrt{\frac{P}{C}} M\right) t}+\frac{\sqrt{\frac{P}{C}}}{2-\sqrt{\frac{P}{C}} M} \epsilon_{t h} .
$$

This expression can be directly compared with measurement. 


\section{Measurement Results}

Both transverse emittances were measured at the end of the cooling cycle for a range of stochastic cooling power levels. An emittance measurement was performed in the following way. The power in the desired cooling system was set at a given value, the beam was injected and the cooling was turned off after 2.3 seconds. The emittance was then measured by using the scrapes (see Appendix). This procedure was repeated at a new value of the power, while the power in the other system was kept at the constant setting. These measurements were done in both transverse cooling systems at the intervals of about 100 Watts from 0 to 1500 Watts. The results are shown in Figs. 3 and 4 .

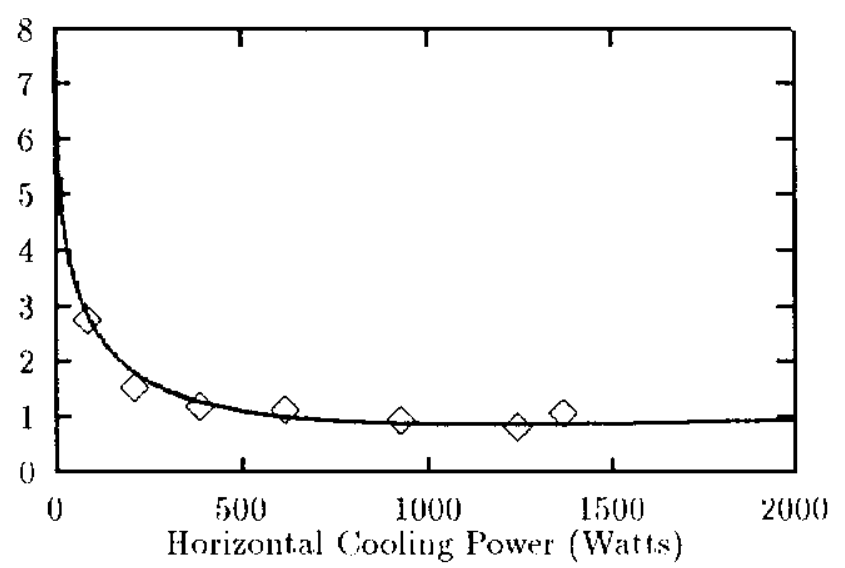

FIGURE 3 The dots are the measured values of the horizontal emittance after 2.3 seconds of cooliug for various values of horizontal power. The power in the vertical system was kejt at 1117 Watts thronghout the measurement. The solid line is the theoretical result, Eq. (7), with $\epsilon_{t h}$ and $C$ as in Eqs. (8) and (10). 


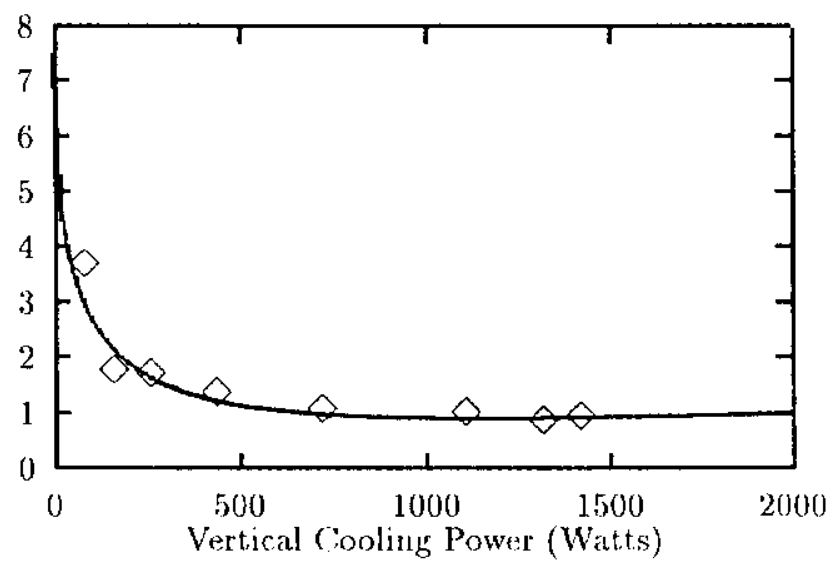

FIGURE 4 The dots are the measured values of the vertical emittance after 2.3 seconds of cooling for various values of vertical power. The power in the horizoulal system wists kept at. 1250 Watts throughout the measurement. The solid tine is the theoretical result, Eq. (7), with $\epsilon_{t h}$ and $C$ as in Eqs. (9) and (10).

From the emittance mesurements the values of $\epsilon_{t h}$ and $C$, were derived by fitting the curve $\epsilon(P)$ at $t=2.3$ seconds to the data. For $M$ the computed value of 10 was used. The best fit is obtained for

$$
\begin{gathered}
\epsilon_{t h}(\text { horizontal })=72.2 \pi \mathrm{mm} \text { mrad } \\
\epsilon_{t h}(\text { vertical })=75.9 \pi \mathrm{mm} \text { mad }
\end{gathered}
$$

and

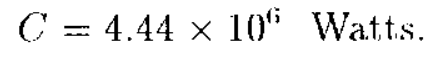

The corresponding curves are shown with the data in Figs. 3 and 4 . Based upon the observed fit, we conclude that the theoretical model, E1. (1), is in excellent agreement with the experimental data. 


\section{Time-Dependence of Mixing}

Due to momentum cooling in the Debuncher, the momentum sprear is reduced by ahout a factor of two during the cooling cycle. Since the mixing factor $M$ is inversely proportional to the momentum spread, it increases by the same factor. How does this time dependence of mixing affert the performance of the cooling system?

The cooling equation (1) now has to be replaced by one with $M(t)=$ $M_{0}\left(1+\frac{t}{t_{c}}\right)$, where $M_{0}=M(0) \approx 10$ is the constant value we used before and $t_{c}$ the duration of the cooling cycle. The new equation is

$$
\frac{d t}{d t}=-g \frac{W}{N}\left(2-g\left(M_{0}+M_{0} \frac{t}{t_{t}}+\frac{\epsilon_{t h}}{\epsilon}\right)\right) t .
$$

It turns out that this equation, too, can be solved exactly, leading to the solution

$\epsilon(g, t)=\frac{2-g M_{0}}{2-g M_{0}\left(1+\frac{t}{t_{c}}\right)}\left\{\left(\epsilon_{0}-\frac{g}{2-g M_{0}} \epsilon_{t h}\right) \epsilon^{-g \frac{W}{N} t\left(2-g M_{0}-\frac{1}{2} g M_{0} \frac{t}{t_{1}}\right)}+\frac{g}{2-g M_{0}} \epsilon_{t h}\right\}$.

As expected, the emittance is going to be larger due to the larger mixing factor. This effect is illustrated clearly in Fig. 5, in which the emittances for the constant and time-dependent mixing are compared for the typical values of the Debundier parameters. It is important to understand the magnitudv of the effect of time rlependent mixing. At the optimal gain, the emitano with a constant mixing factor turns out to be $13 \%$ smalle than with the time dependent one. This is the size of the improvement that can be expected under a proposed scheme ${ }^{3}$ in which the Debuncher lattice is ramped during the cooling cycle such that the mixing factor remains constant. (Note that the change in $\eta$ can offset a change in $\frac{\Delta p}{\eta}$ such that $M$ remains constidnt, Eq.(2).) 


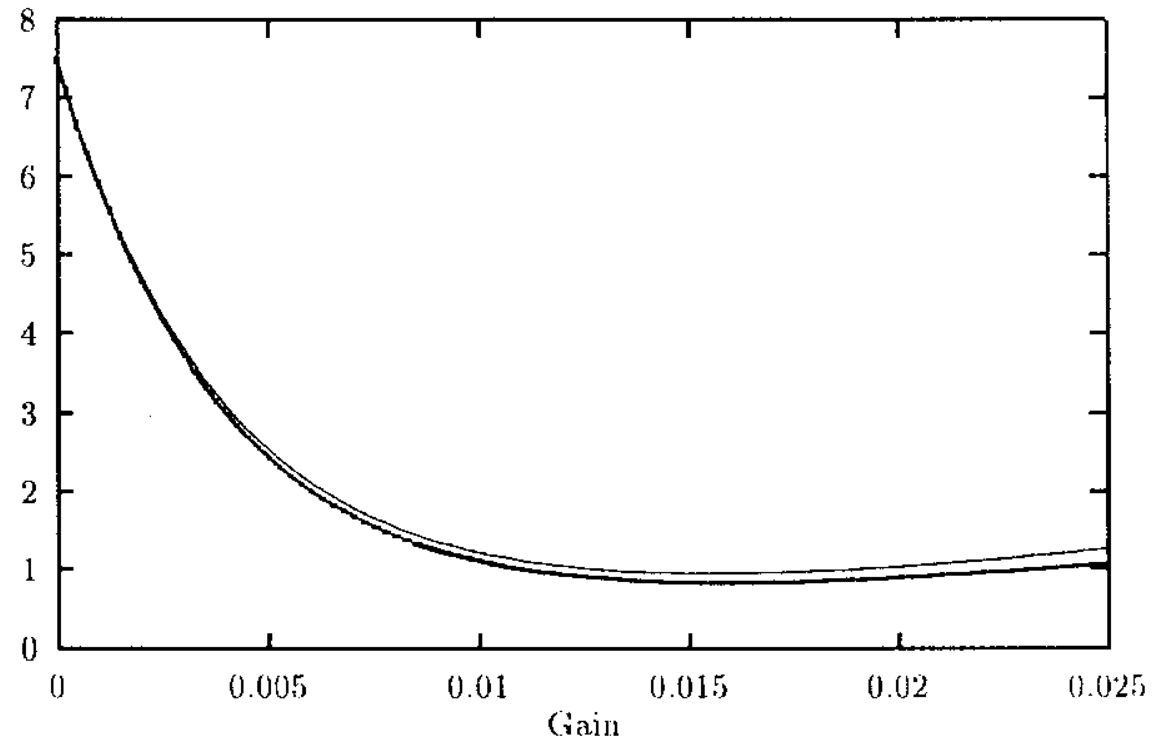

FIGURE 5 The effect of time-dependent mixing. The top curve corresponds to the mixing-factor increase from 10 to 20 during the 2.4 seconds cycle in the Debumcler. The bottom curve is for constant. mixing equal to 10 . 


\section{Gain Shaping}

The optimal gain so far was defined as the optimal constant gain. This gain is, however, optimal only in the average sense, since the cooling is powar limited at the beginning of the cycle and noise limited in the second half of the cycle. We may try to vary the gain in time such that the cooling rate is maximized at every instant throughout the cycle. This is the irlea of gam shaping.

The stochastic cooling system with the gain shaping can be modelled in the following way. From the differential equation (1) we see that the instantaneous cooling rate $\frac{1}{\epsilon} \frac{\partial \epsilon}{\partial t}$ is maximized for gain

$$
g_{\text {opt } t}=\frac{\epsilon}{M \epsilon+\epsilon_{t h}} .
$$

The optimal gain is a function of the emittance and, as we anticipated. increases with the emittance. The differential equation for the time evolution of the emittance with this optimal gain is

$$
\left(M+\frac{\epsilon_{t h}}{\epsilon}\right) \frac{d t}{\epsilon}=-\frac{W}{N} d t .
$$

This equation can be solved analytically, albeit only in the implicit form

$$
M \ln \frac{\epsilon_{0}}{\epsilon}+\frac{\epsilon_{t h}}{\epsilon}=\frac{W}{N} t+\frac{\epsilon_{t h}}{\epsilon_{0}}
$$

This, of course, can be solved mumerically (see Fig. (6), lnt even without that we can see that the emittance is an ever decreasing function of time:

$$
\dot{\epsilon}=-\frac{W \epsilon^{2}}{M \epsilon+\epsilon_{t h}}
$$

which is negutioe for all times $t$. For long cooling times the emittance goes to zero (and not to a finite value)

$$
t_{\infty}=0
$$

as can be directly verified from (13).

The emitance as a function of time is shown in Fig. (i and the corresponding gain shaping in Fig. 7. 


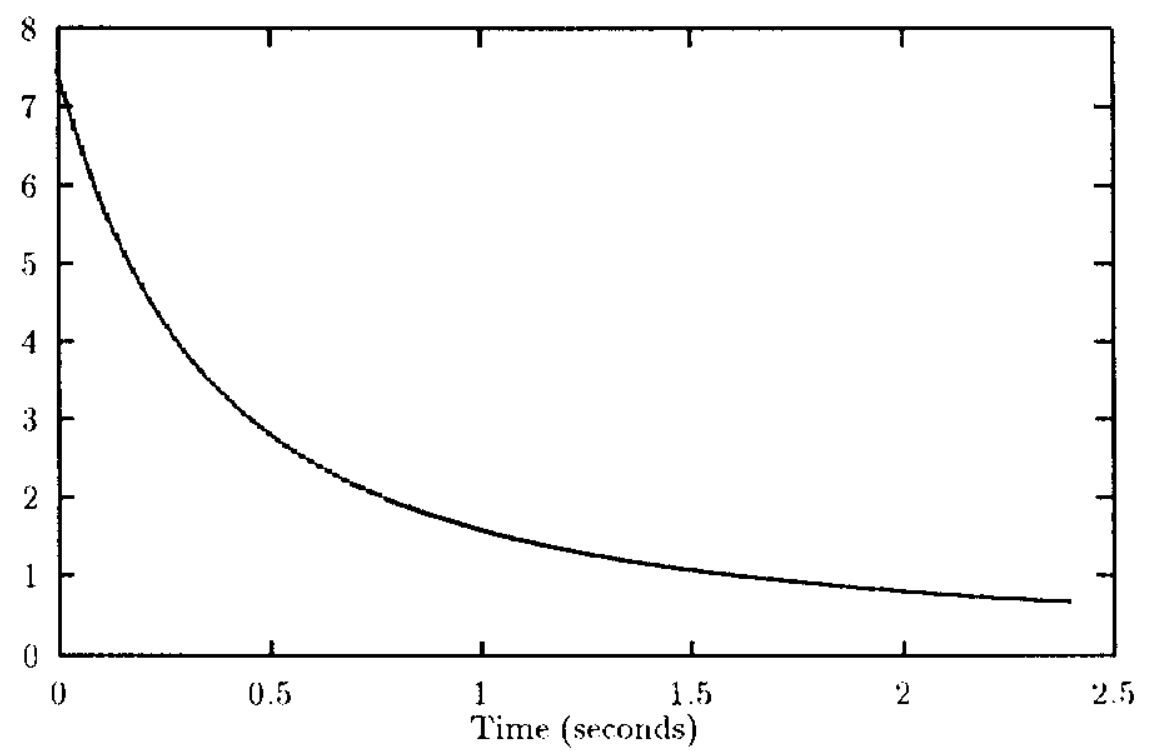

FIGURE 6 The horizontal emittance as a function of time for ideally slapesl gain The emittance approaches zero and not a finite value.

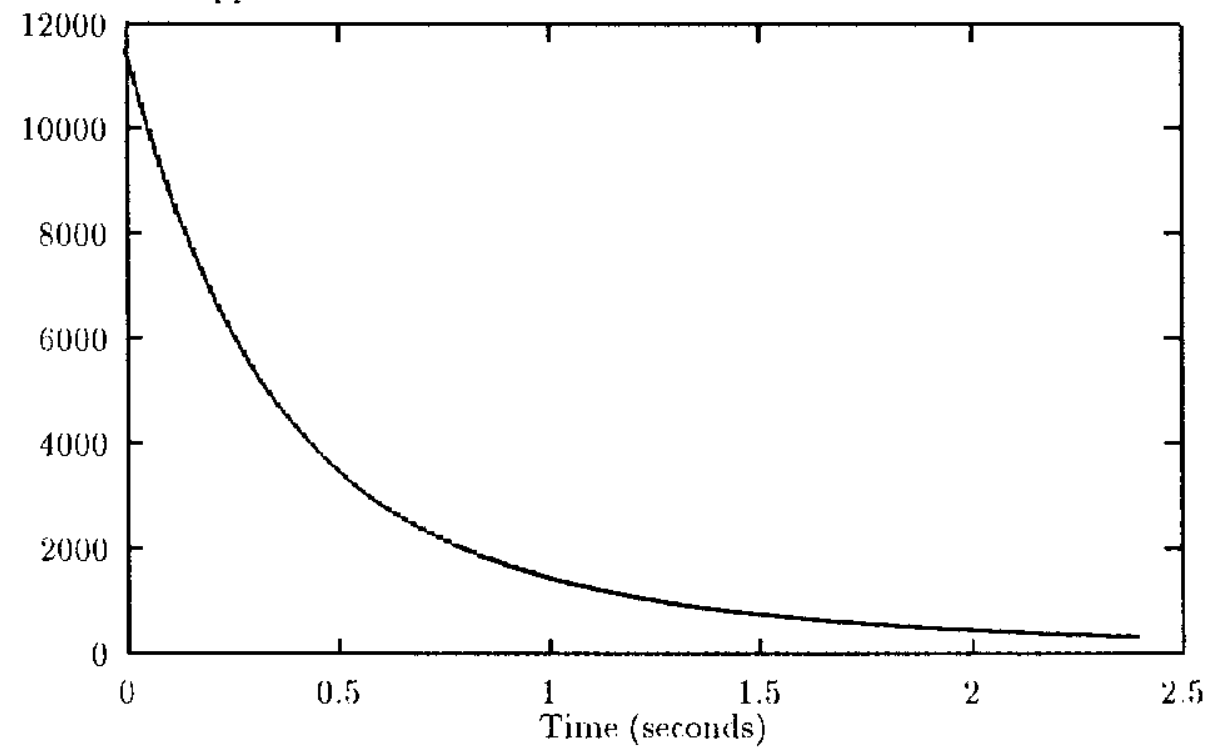

FIGURE 7 The ideal gain shaping for the Debuncher with the present values of parameters. The average power is 2277 Watts. 


\section{Smart Pickups and Kickers}

Pickups and kickers can be made such that their gap increases or deneases proportional to the beam size (i.e. to the square root of the emittance). In the Debuncher, this would increase the signal-to-noise ratio up to a factor of seven - a dramatic improvement, particularly relevant for the Main Injector era, as will be discussed in Section 7 . Consider an example of a typical improvement of the Debuncher performance under the proposed scheme. If the present Debuncher were equipped with smart pickups and kickers, a final emittance of $1 \pi \mathrm{mm}$ mrad could be achieved with only 350 Watts per plante which is less than at thind of the present power, Fig. 8 .

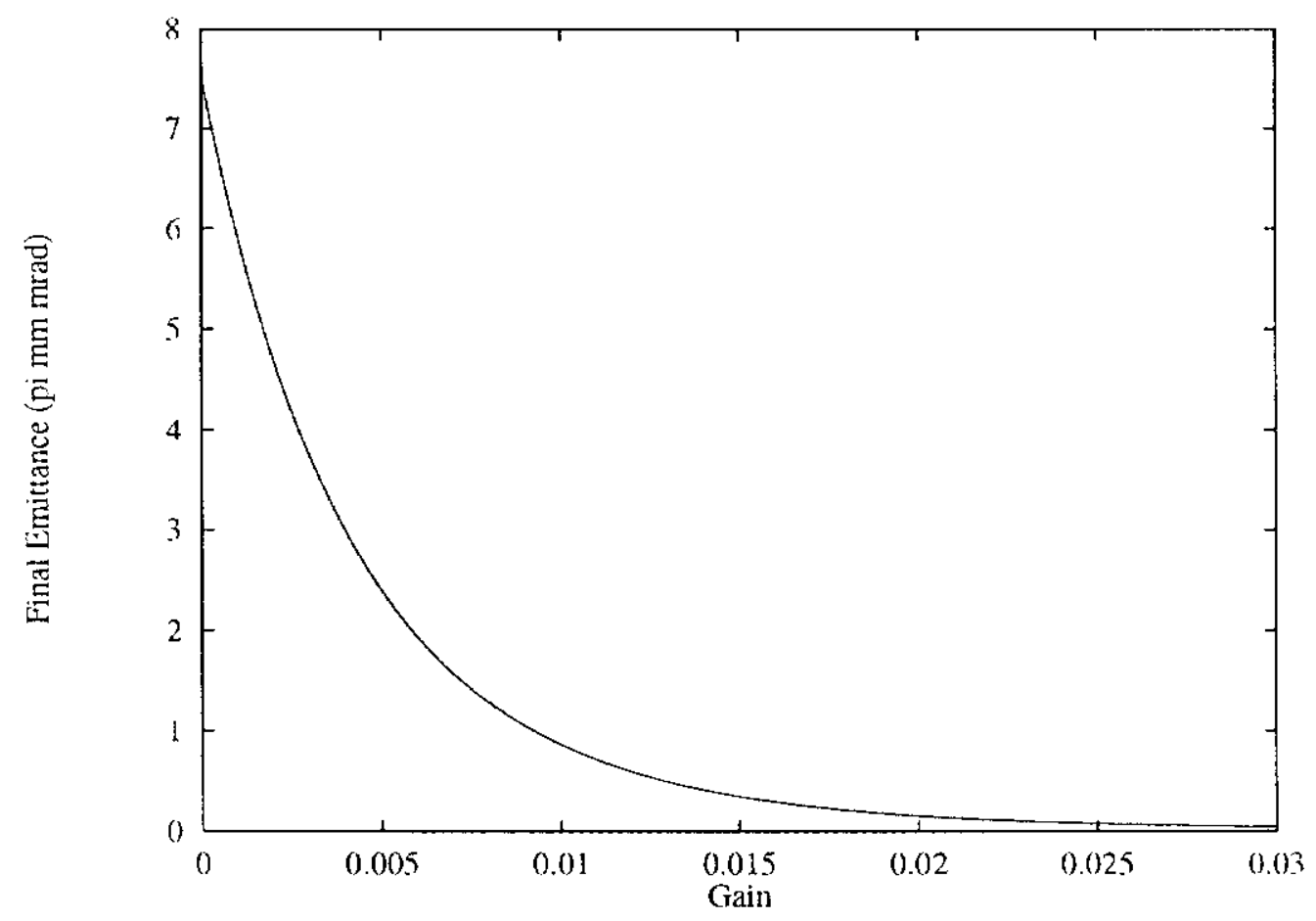

FIGURE 8 Final emit.tance vs. gain plot for the present. Debuncleer stochastic cooling system equipped with smart pickups and kickers. The final emittances of $1 \pi \mathrm{mm}$ mirat can be achieved with 350 Watts per plane, less than a thircl of the present power. (ITise gain-to-power conversion from Section 3.) 
By ruming at 1500 Watts, the enittance wonld reach $0.2 \pi \mathrm{mm}$ mad at the end of the 2.4 seconds cycle, Fig. 9.

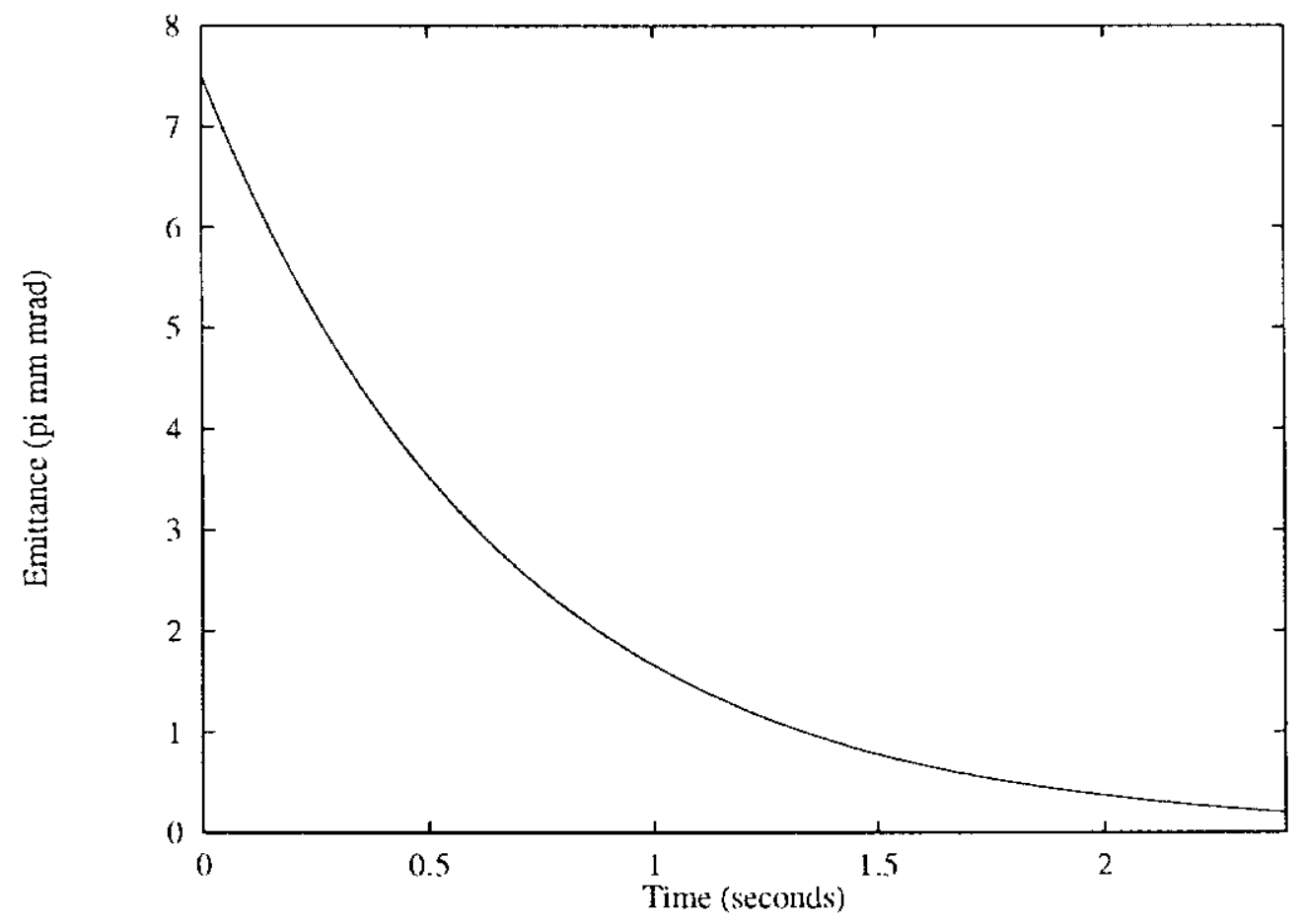

FIGURE 9 Time evolution of emittance with the present. Debuncher stochastic cocling system equjpeped with smart pickups and kickers and run at the constant power of 1500 Watts.

Here it was assumed that the motion of pickups and kickers fully compensates the emititance dependence of the signal-to-noise ratio. In that case the term $\epsilon_{t h} / \epsilon$ in $\mathrm{Eq}_{\mathrm{l}}$ (1) is constant and the optimal gain is also constant. One can also envisage a system in which the needed compensation is partly achieved by using the smart pickups and kickers and partly by using the gain shaping. As wjll be pointed out in Section 8 , the smart pickup and kicker proposal will be a strong candidate amongst the design possibilities in the Main Injector era. 


\section{Debuncher in Main Injector Era}

As mentioned before, in the Main Injector era there will be a threefold increase in the beam intensity in a $40 \%$ shorter cooling cycle. It is interesting to study the performance of the present stochastic cooling system under these new conditions. The time evolution of the emittance is shown in Fig. 10. As can be seen clearly from the figure, the value of the emittance after 1.5 seconds is approximately four times larger than permissible.

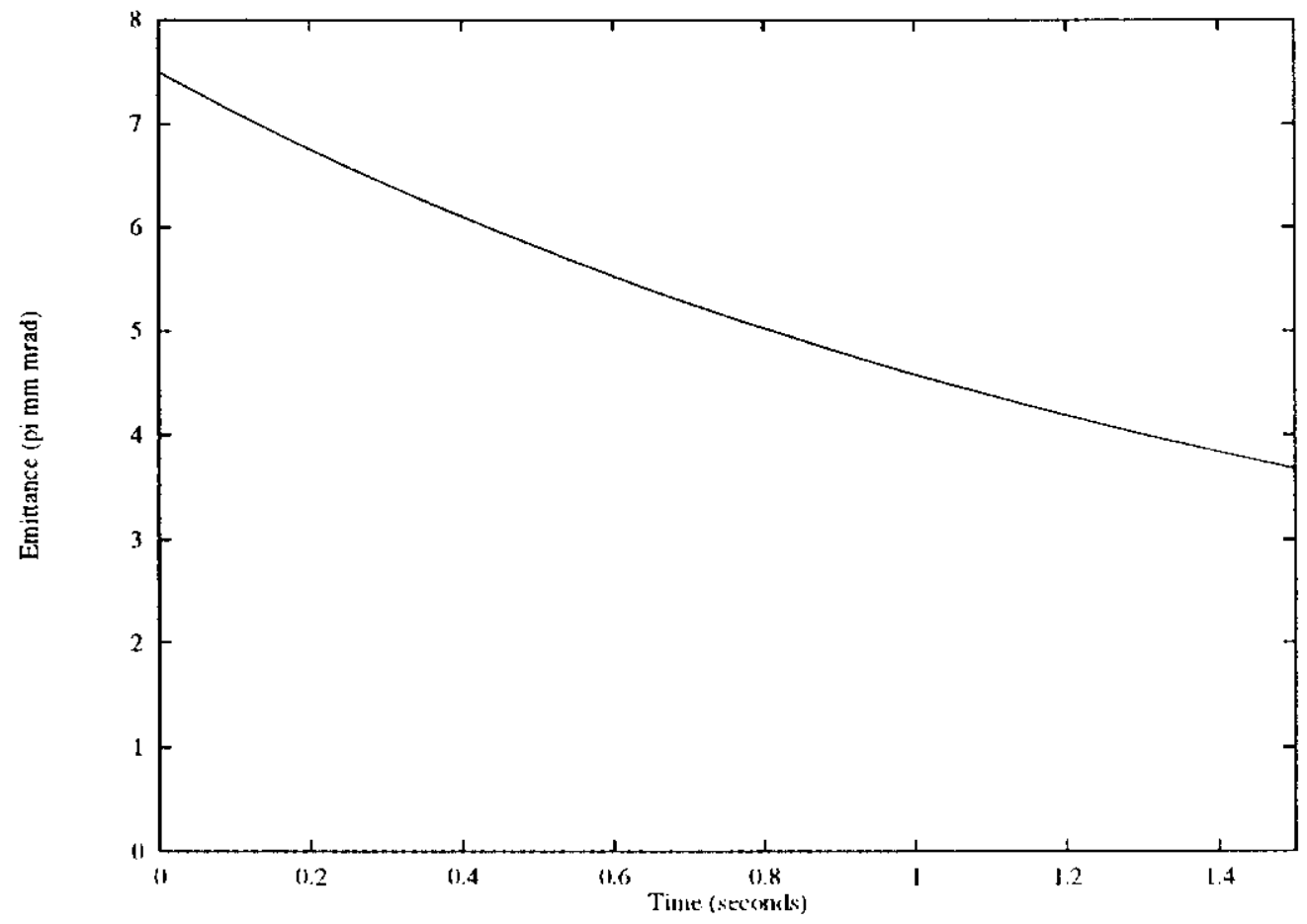

FIGURE 10 The horizontal enittance in the Debuncher with the Main-Injector-erta beam and with the present stochastic cooliug system rum at 2000 Watts (present. maximum) 
The list of upgrade options includes:

1. Increase in cooling power.

2. Gain shaping.

3. Cooling of the resistors to $20 \mathrm{deg}$. Kelvin (gaseous helium).

4. Smart pickups and kickers.

5. Increasing the bandwidth to $4-8 \mathrm{CHz}$.

Any upgrade scheme will undoubtedly consist of a combination of several of these. It is of interest therefore to examine the limits of each option. 


\section{Increase in cooling power}

The (horizontal) emittance at the end of the 1.5 seconds cycle as a function of gain is slown in Fig. 11. The model also includes the time dependence of mixing (see Section 4). The optimal gain corresponds to 6100 Watts per plane, achieving the emittance of $3 \pi \mathrm{mm}$ mrad at the end of the cycle.

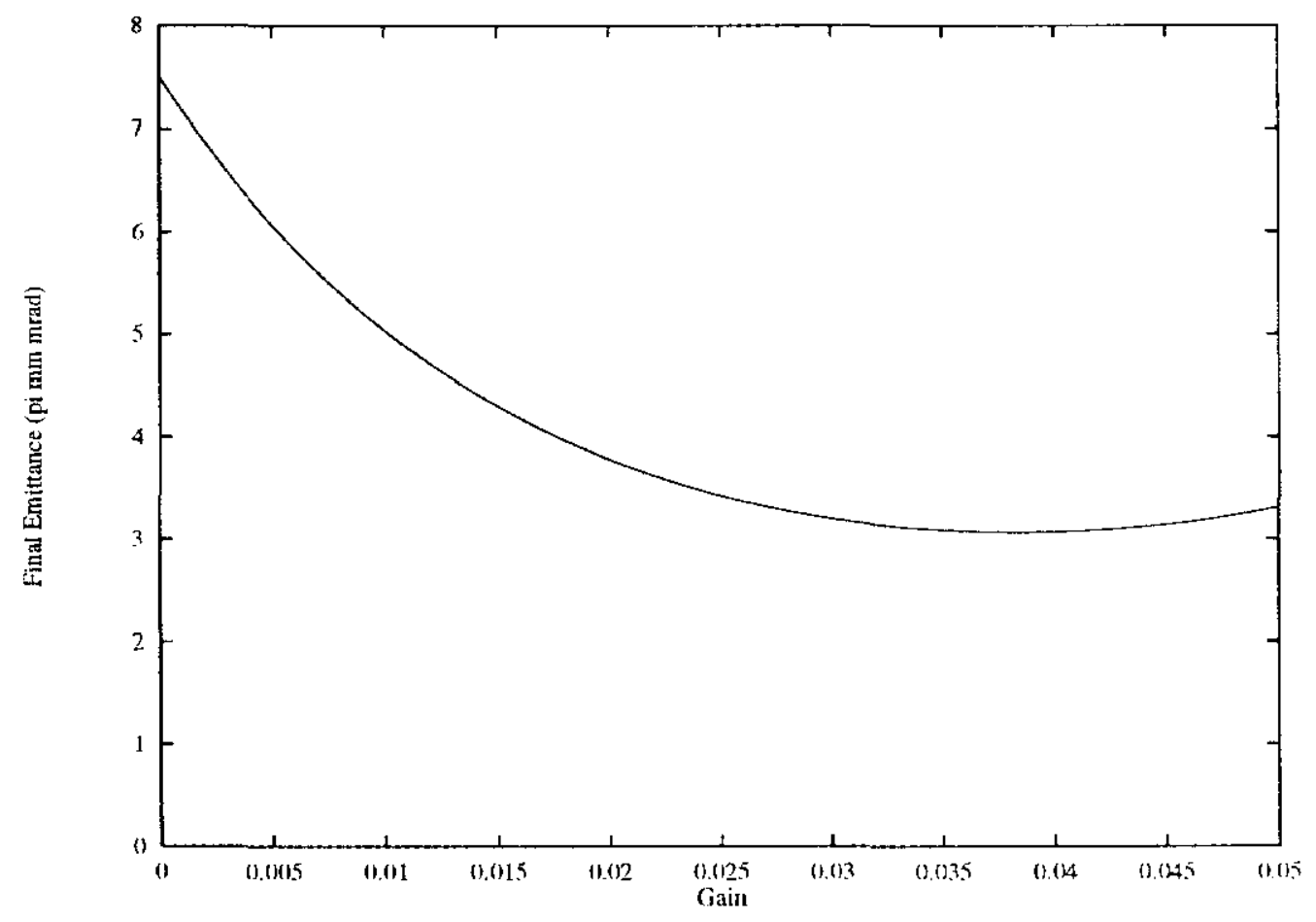

FIGURE 11 The torizontal emittance in the Debuncler as a function of gain. The optimal gain corresponds to 6100 Watt.s. 


\section{Gain shaping}

The gain shaping would lower the emittance to $1.8 \pi \mathrm{mm}$ mrad but requires enormous peak power of 25 kilowatts per plane at the begimning of the cycle. The average power is 15 kilowatts.

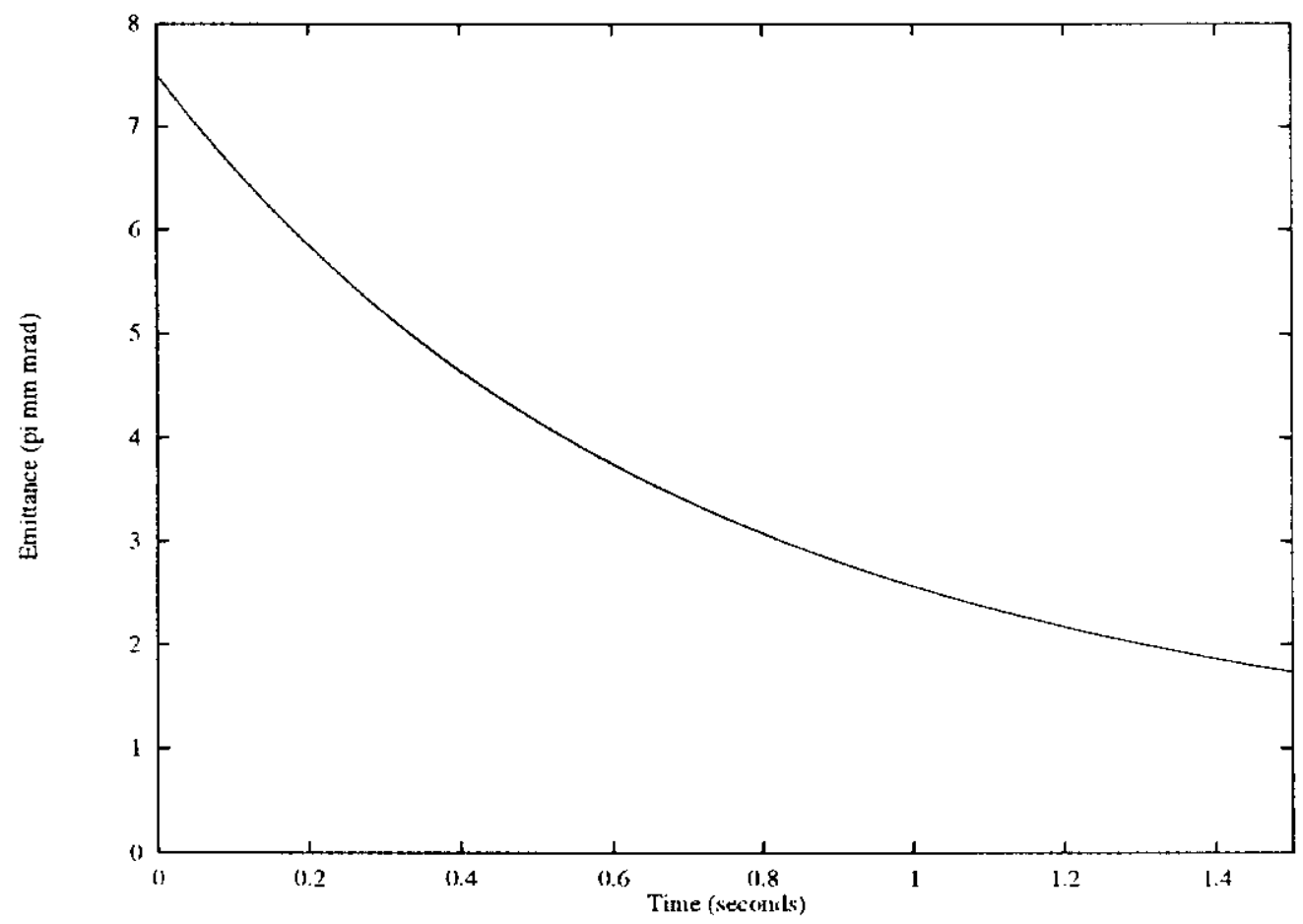

FIGURE 12 The time development of the horizontal enittance with the eptimally shaped grain. The final emittance is $1.8 \pi \mathrm{mm}$ mrand. 


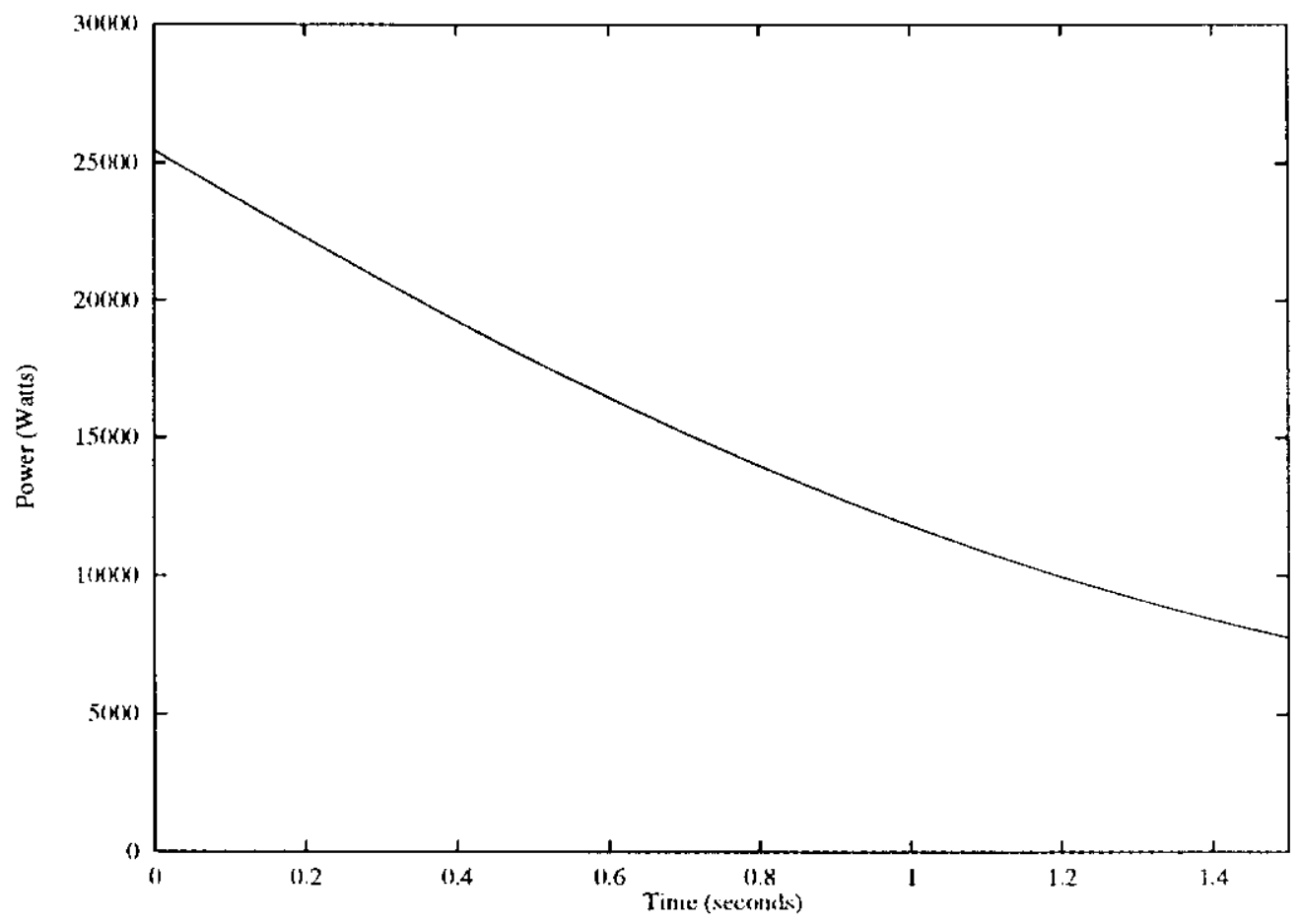

FIGURE 13 The optimal shape of the power whicl produces the emittance of Fig. 12. The average power is ahout 15 kilowat.ts. 


\section{Cooling the resistors}

Cooling the resistors to $T=20^{\circ} \mathrm{K}$ (instead of present $80^{\circ} \mathrm{K}$ ) will decrease" $\epsilon_{t h}$ by a factor

$$
\frac{40+80}{40+20}=2,
$$

cf. Eq. (4). The final emittance is reduced by only an insignificant amount, as can be seen in Fig. 14. If combined with increased power, the enittance can be further reduced to about $3 \pi \mathrm{mm}$ mrad as shown in Fig. 15. It turns out, however, that this improvement is not sufficient for the Main Injector era, unless one ases very high bandwidth (e.g. $8-16(\mathrm{GHz})$ amplifiers, as discussed in Section 8.

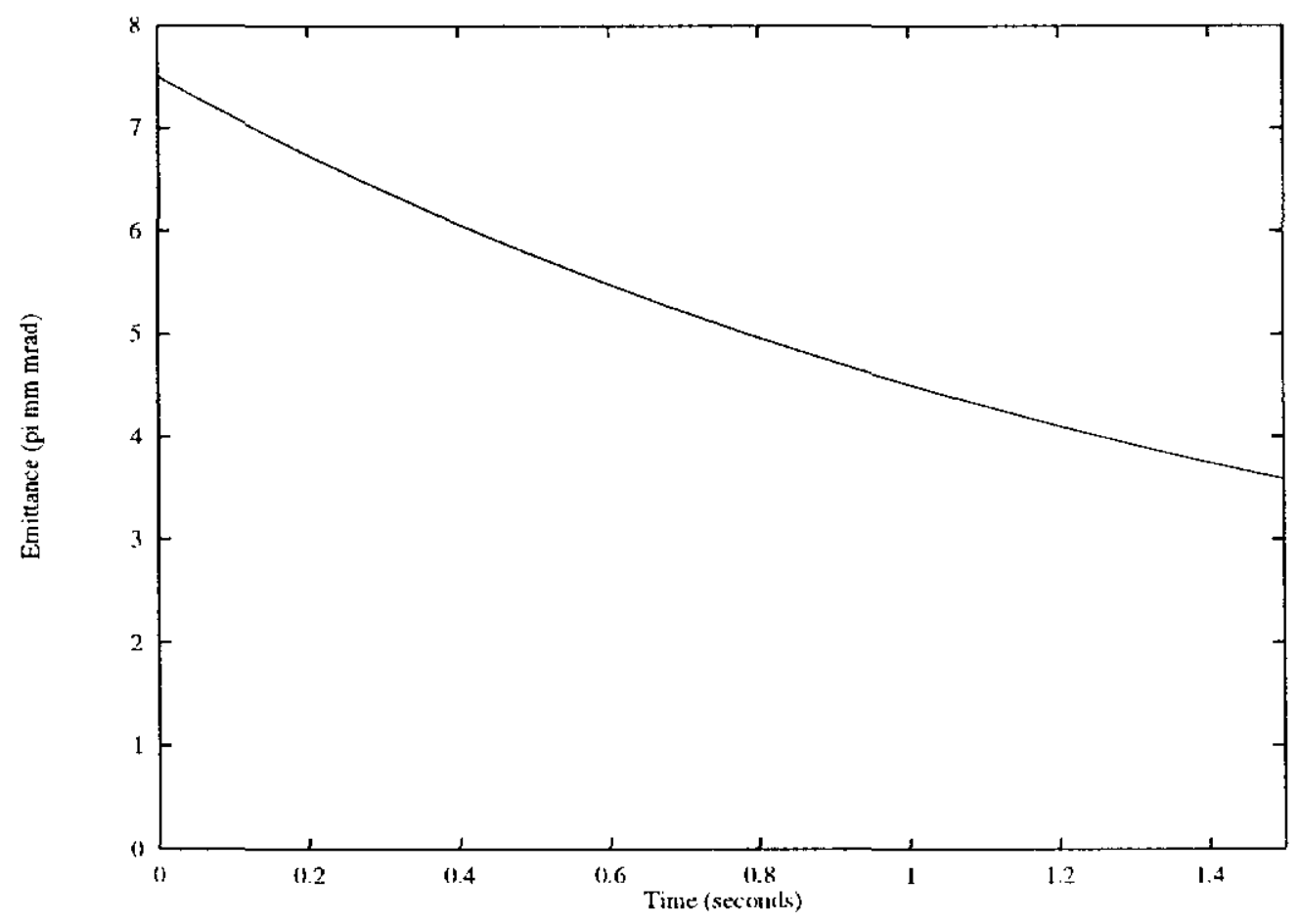

FIGURE 14 Time evolution of the emittance with $T_{F}=200^{11} \mathrm{~K}$. Compare with Fig 10.

The optimal gain is again obtained from the emittance-gain plot, Fig. 15. 


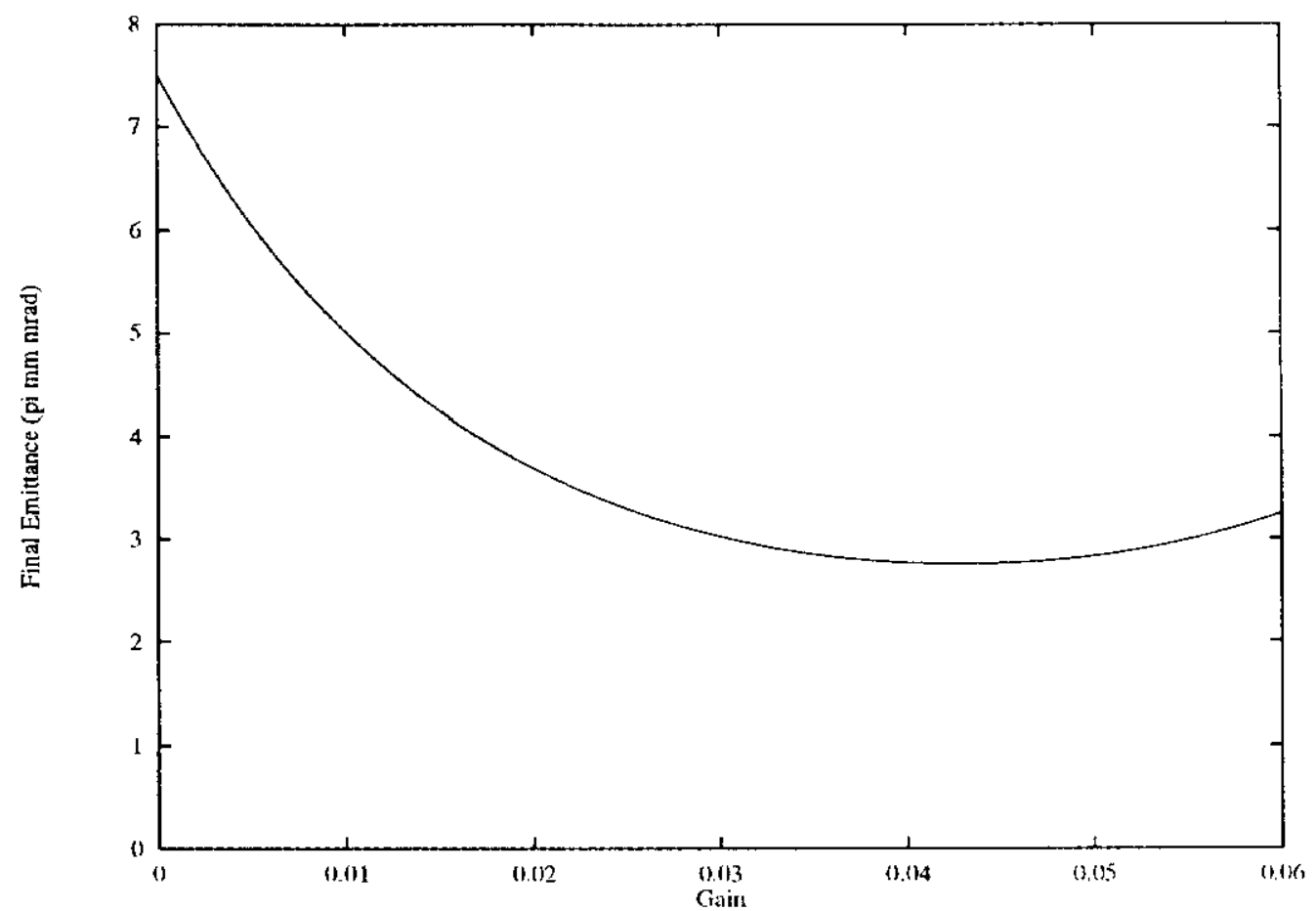

FIGURE 15 Final emittance as a function of gain with cold resistors (20" $\mathrm{K}$ ). The optimal power is alsout. 7500 Walts. 


\section{Increase the bandwidth}

Doubling the bandwidth to $4 \mathrm{GHz}$ doubles the cooling rate. The optimal constant power turns out to be 4800 Watts per plane, leading to the emittance of $1.5 \pi \mathrm{mm}$ mrad, Fig. 16 .

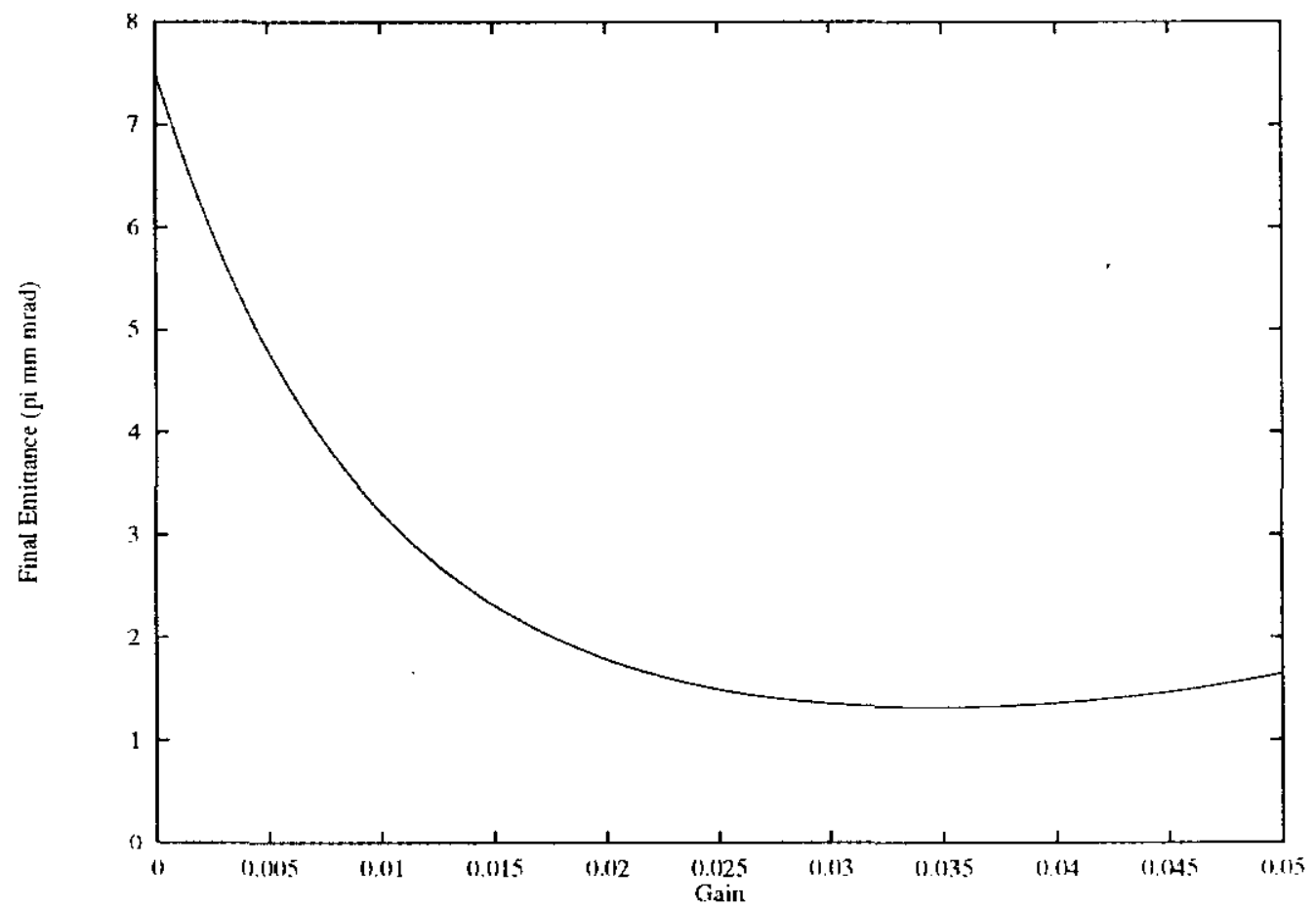

FIGURE 16 Emittance s. gain with $W=4 \mathrm{GHz}$. 


\section{Smart pickups and kickers}

Smart pickups and kickers offer dramatic improvement in achievable emittances. Actually, they are the only possibility if we do not contemplate exotii bandwidths. The resulting emittance ns. gain curve is given in Fig. 17. The optimal power is about 25 kilowatts and the final emittance $1.2 \pi \mathrm{mm}$ mrad. Note that by running at only 2000 Watts the emittance is only larger by a factor of 2.5, Fig. 18. This can easily be reduced to $1 \pi m m$ mrad by using higher bandwidth as will be shown in Section 8 .

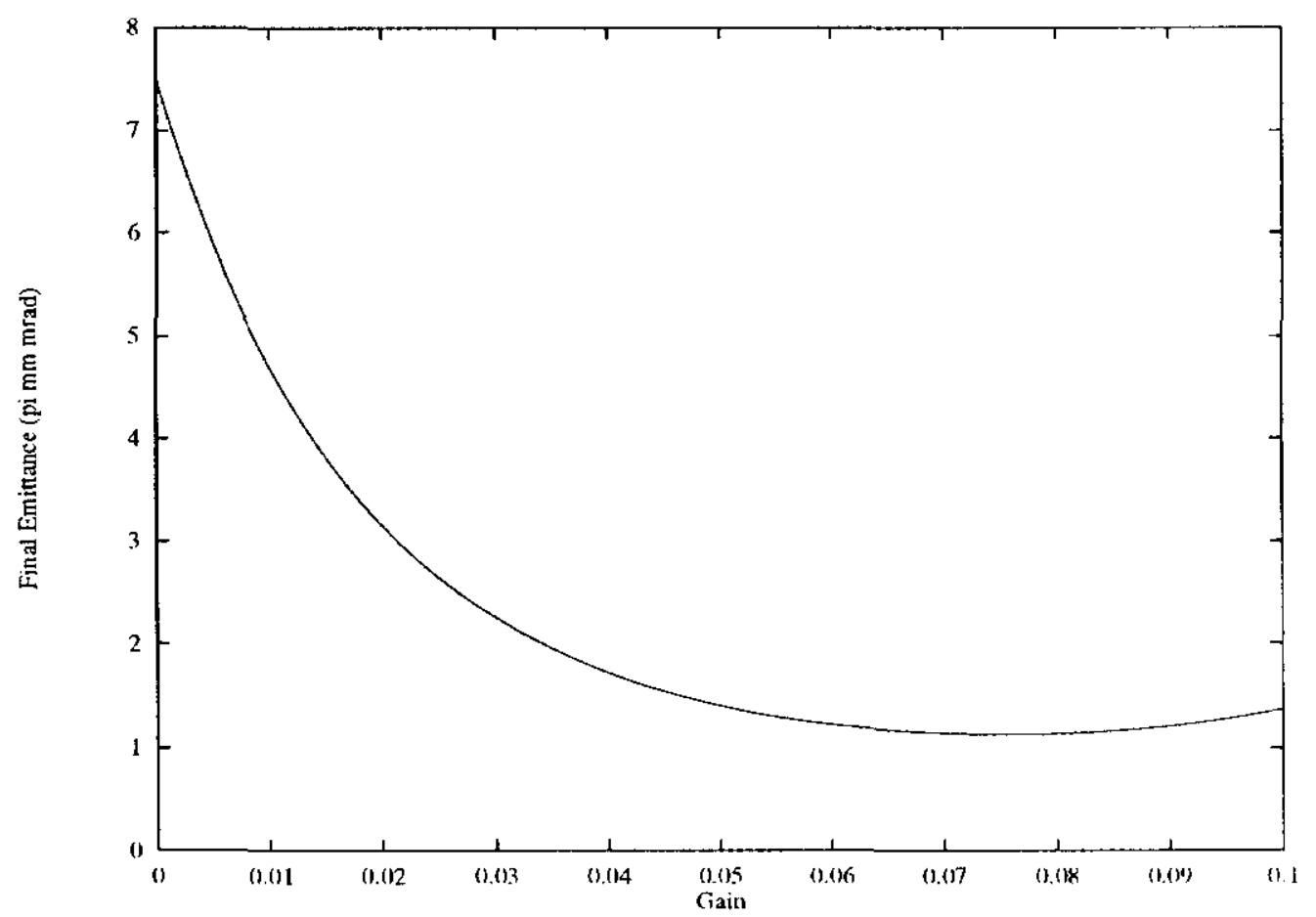

FIGURE 17 Enittance vs. gain plot for Main Injector era beam with smart pickups and kickers. The optimal power is about 25 kilowatts. 


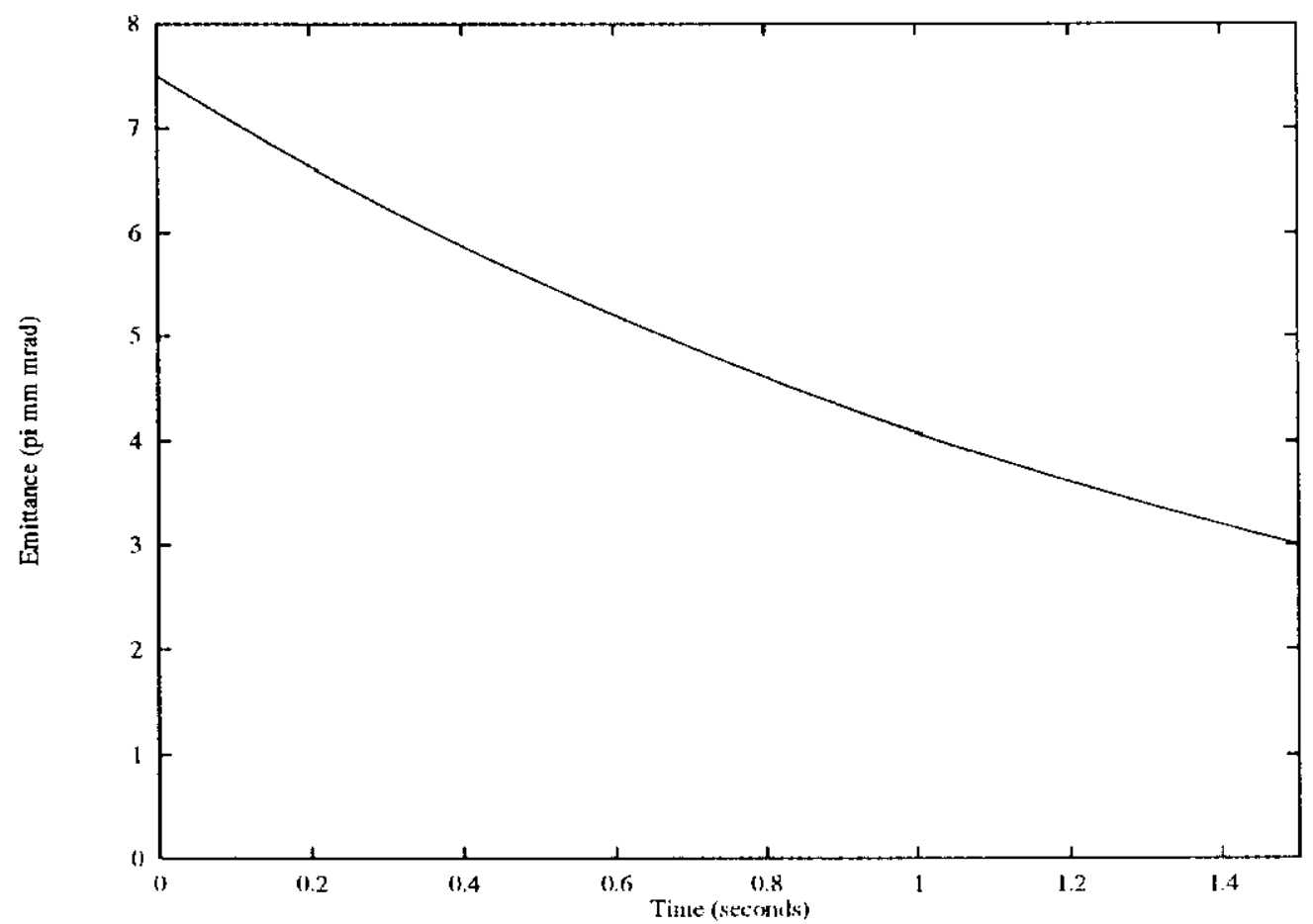

FIGURE 18 The time evolution of emittance with smart pickups and kickers run at the presently maximal power of 2000 Watts. 


\section{A Design for the Main Injector Era}

The present study suggests that the final emittance of the order of $1 \pi$ mm mrad can only be achieved with the bandwidth increase accompanied by a signal-to-noise enhancement, such as smart pickups and kickers or cold resistors. It is important to point out that doubling the bandwidth to 4 $\mathrm{GHz}$ effectively exchudes the option of cold resistors as the required power is impractically high. The emittance in this case cannot be lowered to $1 \pi$ mm mrad with less than about 6000 Watts per plane, as shown in Fig. 19. If one chooses a higher bandwidth, the possibility of using the cold resistors becomes again viable. Therefore, it appears that the smart pickups and

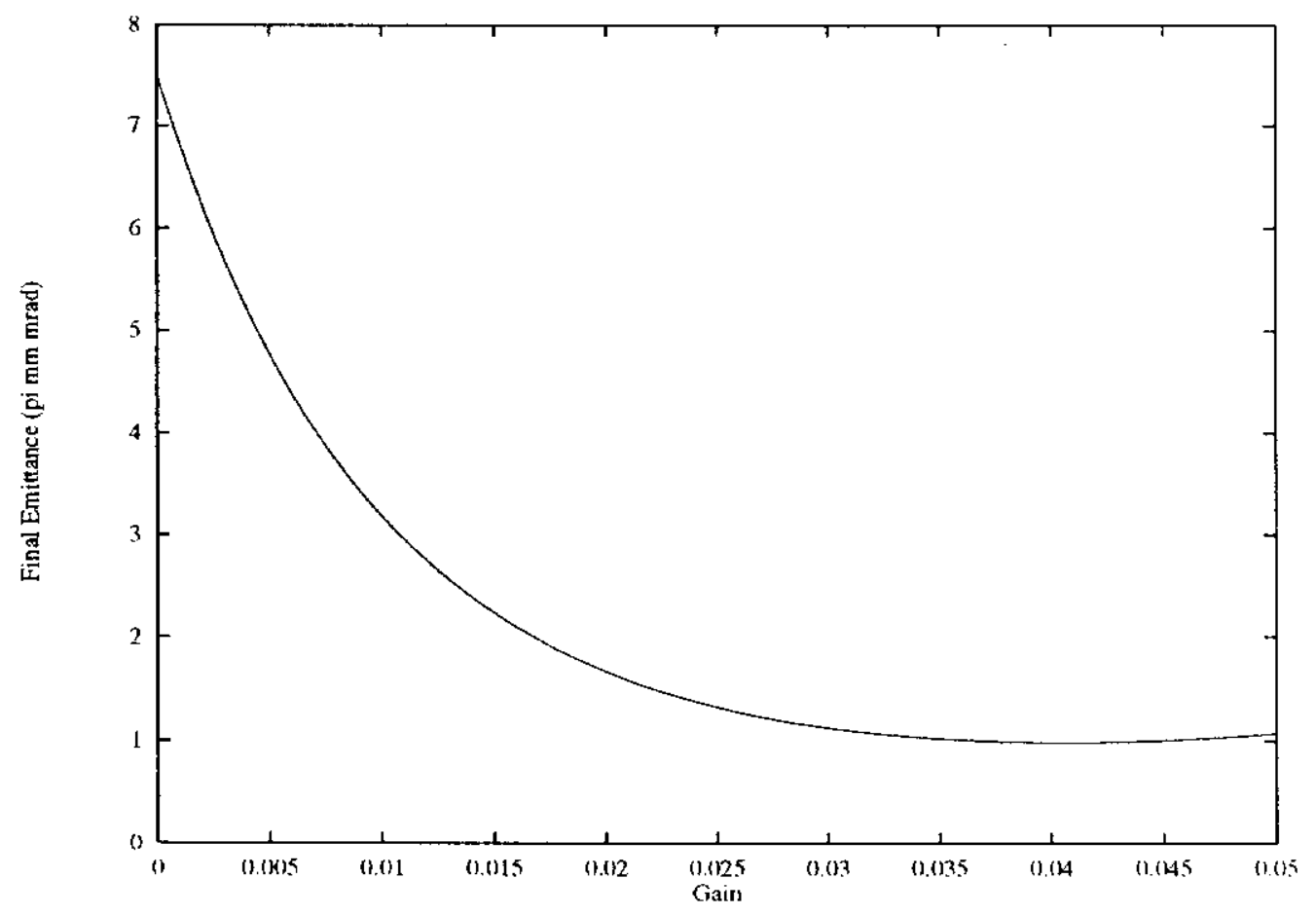

FIGURE 19 Emittance $v$. gain for $W=4 \mathrm{GHz}$ and cold resistors. Tle smallest. achievable emittance is $1 \pi \mathrm{mm}$ mrad at power level of 6000 Watts per plane.

kickers remain as the only possibility if we do not wish to nse bandwirtts higher than 4-8 $\mathrm{GHz}$ and power higher than abont 3000 Watts in each plane. As an example, if the pickus and kickers are constructed with the gal varying between 1.1 and $3 \mathrm{~cm}$ the system would have a constant signal-to-noise 
ratio throughout the cycle and the optimal gain shape would be constant. The calculation shows that warm pickups and kickers provide satisfactory performance, thus there is no need to build smart and cryogenic pickups and kickers which would be technologically much more difficult to realize. The outline of a design of a viable system and its calculated performance are given in the Table 1 and the corresponding emittance ns time plot in Fig. 20.

\begin{tabular}{|c|c|}
\hline PARAMETER & VALUE \\
\hline Amplifier bandwilth & $4 \mathrm{GHz}$ \\
Maximal power & 2800 Watts per plane \\
Pickup/kicker gap & variable $1.1-3.0 \mathrm{~cm}$ \\
Pickup temperature & $80+40{ }^{\circ} \mathrm{K}$ \\
Final emittance & $1 \pi \mathrm{mm}$ mrad \\
\hline
\end{tabular}

TABLE 1 The hasic parameters of the betatron cooling system for the Main Injector era.

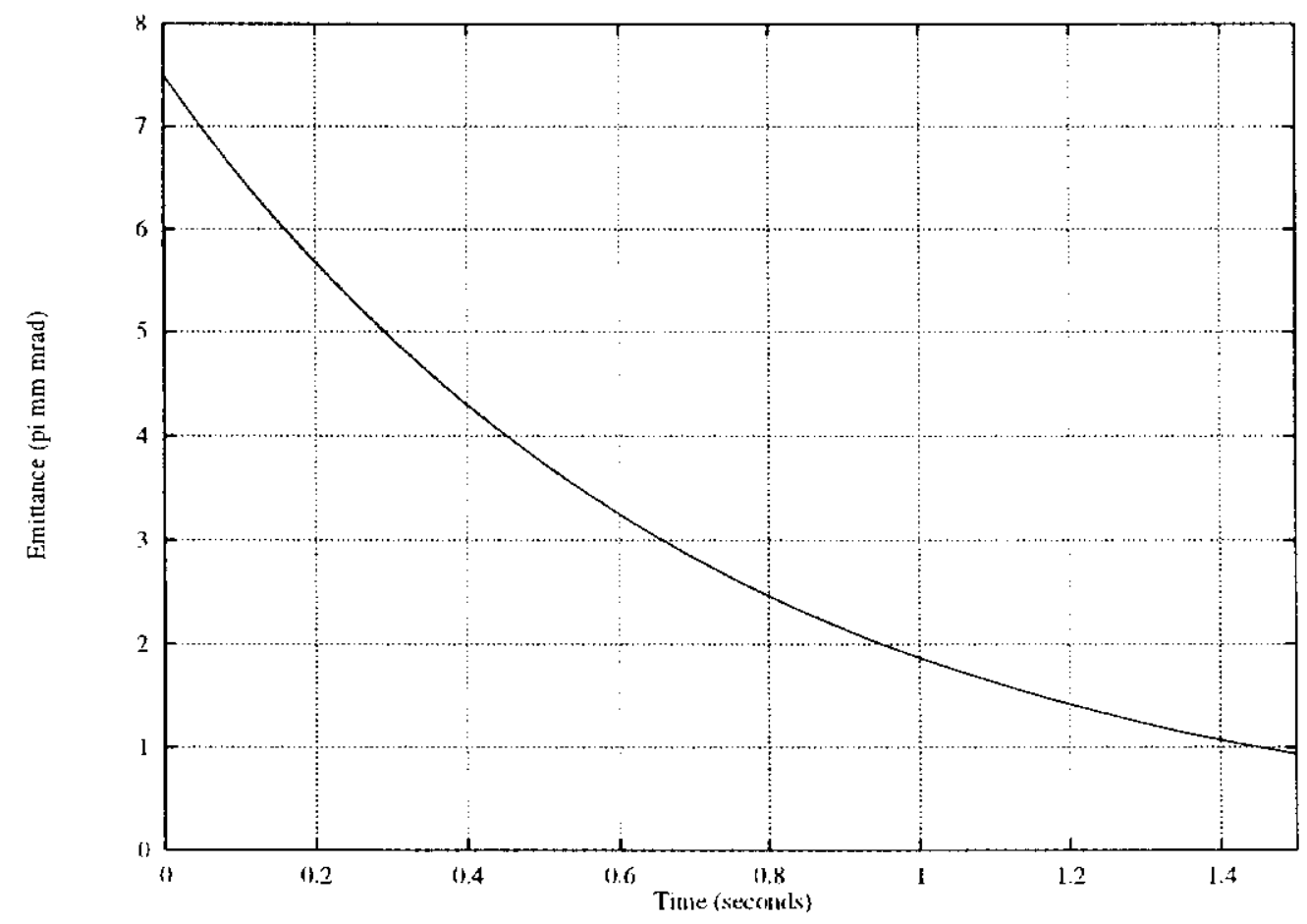

FIGURE 20 Enittance ws, time for $W=4(\mathrm{iHz}$ and smate pickups and kickers. The power is 2800 Watts per plane. 


\section{Appendix}

\section{Measuring Transverse Emittance by Using Scrapers}

Consider the beam with a. Gaussian ristribution in phase space:

$$
\rho\left(x, x^{\prime}\right)=\frac{\beta}{2 \pi \sigma^{2}} e^{-\left(x^{2}+\left(\beta x^{\prime}+\alpha x\right)^{2}\right) / 2 \sigma^{2}} .
$$

The rms emittance of the beam is defined as $t=\sigma^{2} / \beta$. How does one determine the rms emittance from the scraper measurement? As the scraper moves toward the center of the beam, the outer layers are "peeled off" as shown in Fig. 21. The measured beam cument is proportional to the

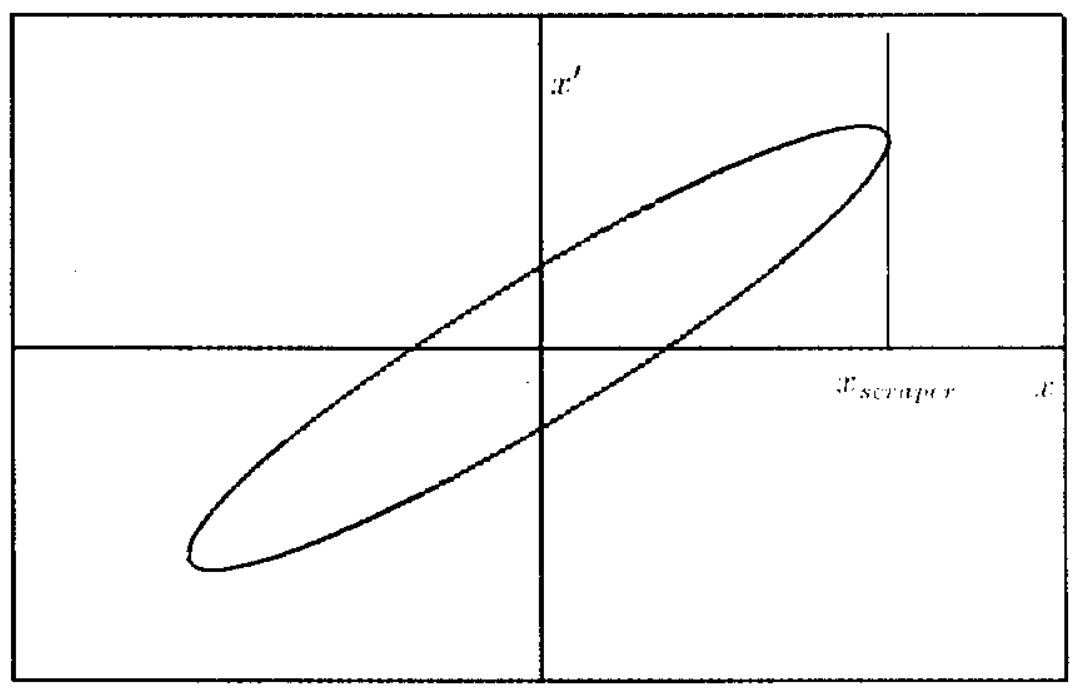

FIGURE 21 The beam ellipse defined by the scraper position.

fraction of the bean insirle the aperture defined by the scraper position $x_{s:}$ :

$$
\begin{gathered}
f=\frac{\beta}{2 \pi \sigma^{2}} \int_{e l l i p_{1}, x e} d x d x^{\prime} \epsilon^{-\left(x^{2}+\left(\beta x^{\prime}+(r s)^{2}\right) / 2 \sigma^{2}\right.} \\
f=1-\epsilon^{-x^{2} / 2 \sigma^{2}}
\end{gathered}
$$


The fractional beam current as a function of $x_{s} / \sigma$ is shown in Fig. 22. This is to be compared with a typical measurement shown in Fig. 23.

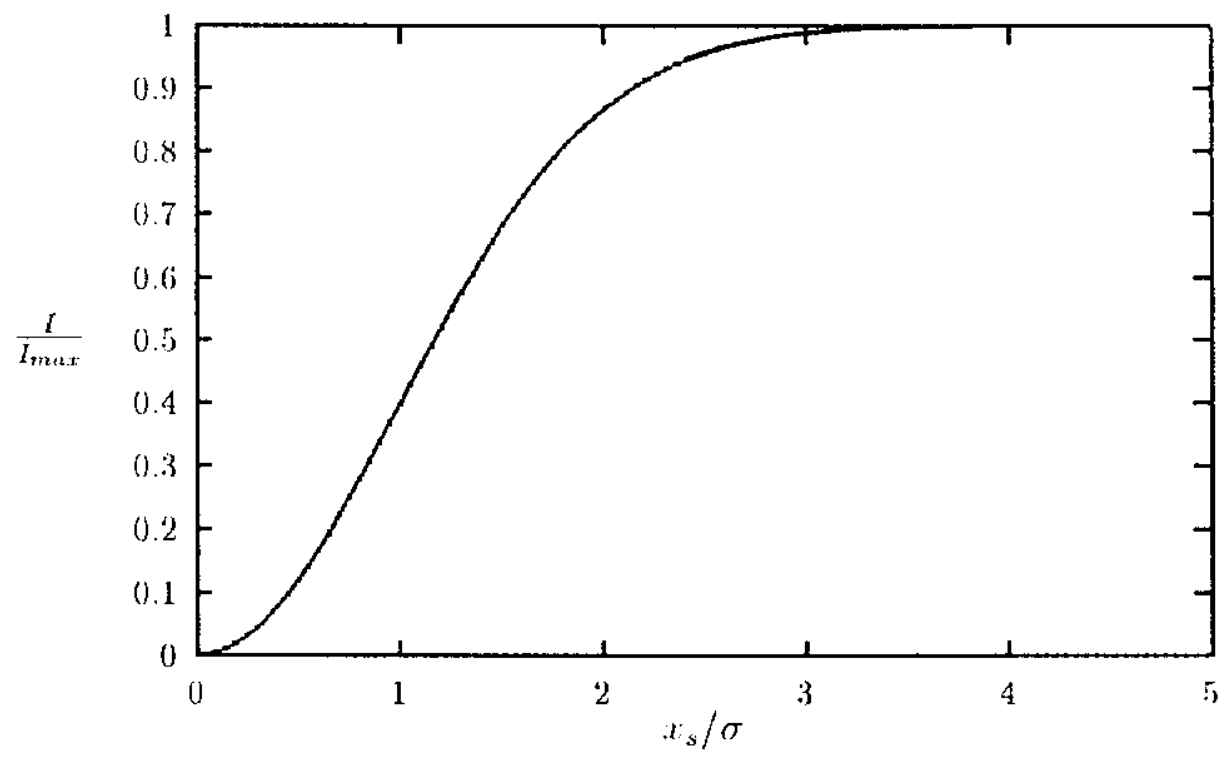

FIGURE 22 Beam current $v$ s. scrajeer position for Gaussian bean.

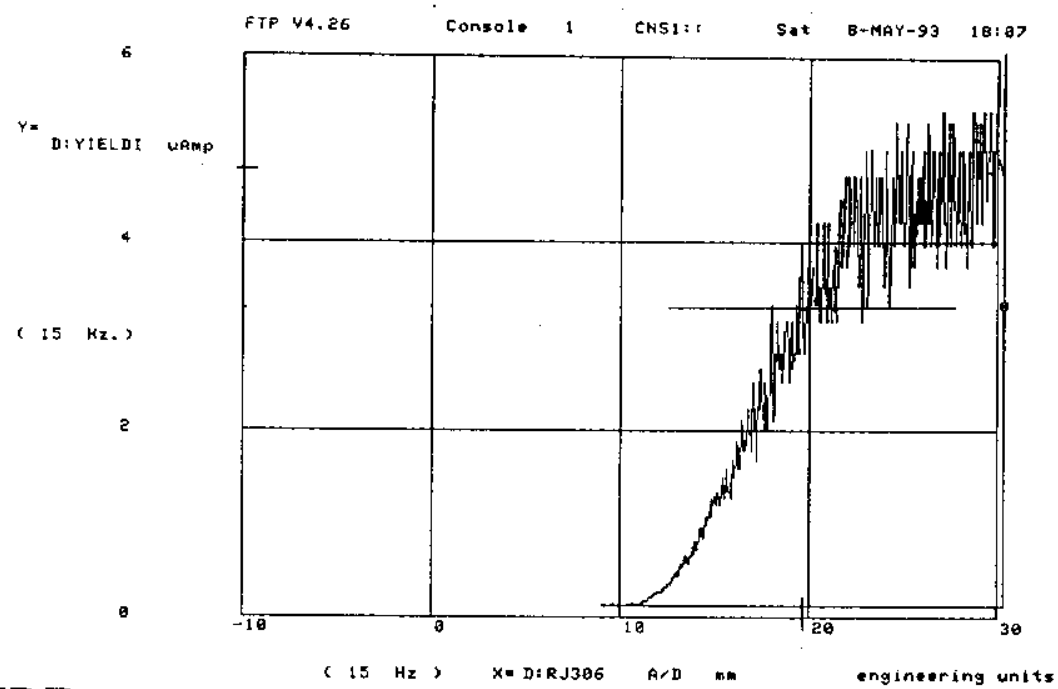

FIGURE 23 Bean current $v$ s. scraper position * measurement data. 
The goal is to obtain the $\sigma$ of the distribution as a function of $x_{s}$ and $f$,

$$
\sigma^{2}=\frac{x_{s}^{2}}{2 \ln \frac{1}{1-f}}
$$

The rms emittance is then

$$
\epsilon=\frac{x_{s}^{2}}{2 \beta \ln \frac{1}{1-f}}
$$

This is the formula used in the measurement of emittance discussed in Section 3. The procedure in these measurements consists of reading $f$ and $x_{s}$ from the data such as those in Fig. 23. Since the $\beta$ function at the position of the scraper is known, the rms emittance is obtained from the above expression.

\section{References}

1 Design Report, Tevatron 1 Project, Fermilals 1984.

${ }^{2}$ V. Visnjic and M. Halling, Proc. Particle Accelerator Conforence 199\%,

${ }^{3}$ M. Church, private communication. 